\title{
A Non-Intrusive Monitoring System for Ambient Assisted Living Service Delivery
}

\author{
Willy Allègre`, Thomas Burger, Pascal Berruet, and \\ Jean-Yves Antoine \\ Lab-STICC (CNRS), European University of Brittany, Université de Bretagne-Sud, \\ Centre de Recherche, BP 92116, F-56231 Lorient Cedex, France \\ E-mail: first.last@ univ-ubs.fr
}

\begin{abstract}
Automation of smart home for ambient assisted living is currently based on a widespread use of sensors. In this paper, we propose a monitoring system based on the semantic analysis of home automation logs (user requests). Our goal is to replace as many sensors as possible by using advanced tools to infer information usually sensored. To take up this challenge, an ontology, automatically derived from a model-driven process, firstly defines user-system interactions. Then, the use of rules allows an inference engine to deduce user location and intention leading to adapted service delivery.
\end{abstract}

Keywords: Pervasive Systems, Ambient Assisted Living, Model-Driven Engineering, Ontologies, Home Automation Services, Context-Awareness

\section{Introduction}

Care for dependents is becoming a major social and economic issue for the next few years. In 2050, 30\% of people from the european countries will be at least 65 years old [1], thus increasing the number of dependent people with chronic diseases. The number of potential caregivers can not evolve accordingly, so that home automation for ageing and disabled people is considered as an alternative solution to fill the gap, allowing one to facilitate or automate devices activation and to provide services tailored to the user needs: we talk about Smart Home.

Due to the miniaturization of electronic devices and their dissemination in the environment, smart home is no longer confined to the simple control of household appliances. This field of application is evolving more and more toward Pervasive Systems [2], where context-awareness is an important challenge. Indeed, knowing who is doing what and where is essential to adapt a "smart behavior", especially in the area of Ambient Assisted Living. The use of sensors distributed in the environment is the most commonly used solution to collect these contextual data. Two main methods can be distinguished, which can be coupled [3-5]: the use of cameras which is intrusive, and the massive deployment of sensors in the environment which implies a significant financial cost.

\footnotetext{
* This work is financed by the Brittany Region and is the result of a cooperation supported by Kerpape MFRRC (http://www.kerpape.mutualite56.fr/).
} 
Previous works from [6] highlighted the interest of monitoring activities based on home automation logs (user requests only) to limit the use of these sensors. Their monitoring system is based on a frequency analysis in which a service is considered as a repeating pattern over time. Based on the assumption of the existence of very regular activities, the goal was to automatically extract and to propose daily living scenarios to wheelchair-bound users with limited capabilities. However, this assumption is not always verified in practice, so that some potential scenarios are not proposed. For example, the user needs to switch on/off lights in rooms leading to the bathroom at night. This kind of interaction can be requested from time to time, in a non-regular manner.

In this paper, instead of a frequency analysis, we propose to use a semantic analysis of each home automation log to interpret service activation at a given time. In this perspective, it is possible to provide the user adapted services without any training steps and using as few sensors as possible. To take up this challenge, we first propose to model user-system interactions and their semantics by means of an ontology (section 2). Then, the use of rules applied to ontology concepts and properties allows an inference engine to deduce user location and intention, leading to service delivery (section 3). Finally, in the context of a large scale deployment, we propose to derive automatically the ontology from a model-driven process developped in previous works (section 4). A real case study (section 5) presents the benefits of the entire work.

\section{An ontology for user-system interaction modeling}

Based on description logics, the ontology described above models the semantics underlying interactions between one user and his environment. As it is not possible to distinguish who activates a switch, we assume that the user stands alone. Obviously, our work should fit the need of a multi-user application, provided each $\log$ can be associated with an individual user id (e.g. services are requested from individual interfaces).

The ontology is made of six fundamental concepts:

- User: someone who interacts with his environment using spread devices

- Device: resource activated by user i) remotely or ii) from the same room

- Basic Service: directly provided by both local and remote devices

- System: interface to collect interactions composed of devices and services

- Location: part of the environment that can contain devices and users

- Effect: effect of services activation on the environment (e.g. luminosity)

This ontology is a useful support to achieve supervision ${ }^{1}$ using as few sensors as possible. From the way the user interacts with his environment, we seek to infer his location and his intention related to the effect produced by services activation. Knowing where the user is and what is his intention, it is then possible to lead to service delivery. This is made possible by the formal semantic of description logics underlying ontologies, but also by rule-based reasoning capabilities.

\footnotetext{
${ }^{1}$ By supervision, we mean not only monitoring, but also service delivery.
} 


\section{From a non-intrusive monitoring to service delivery}

User tracking is an important context-awareness process, from which location can be inferred. In addition to ontology concepts described above, we add semantic relationships to link them. Figure 1 (left side) shows part of the ontology terminology $(T B o x)$ for user location. The Door concept is defined with exactly two links isBetween towards two concepts Room. Given this logic representation, rules are expressed to infer properties such as isLocatedIn, isEntering and isLeaving following basic services activation. Consider for instance the rule 1 from table 1: a user ? $u$ who is using a local activation device ?d which stand in room ? $r$, is himself in this room.

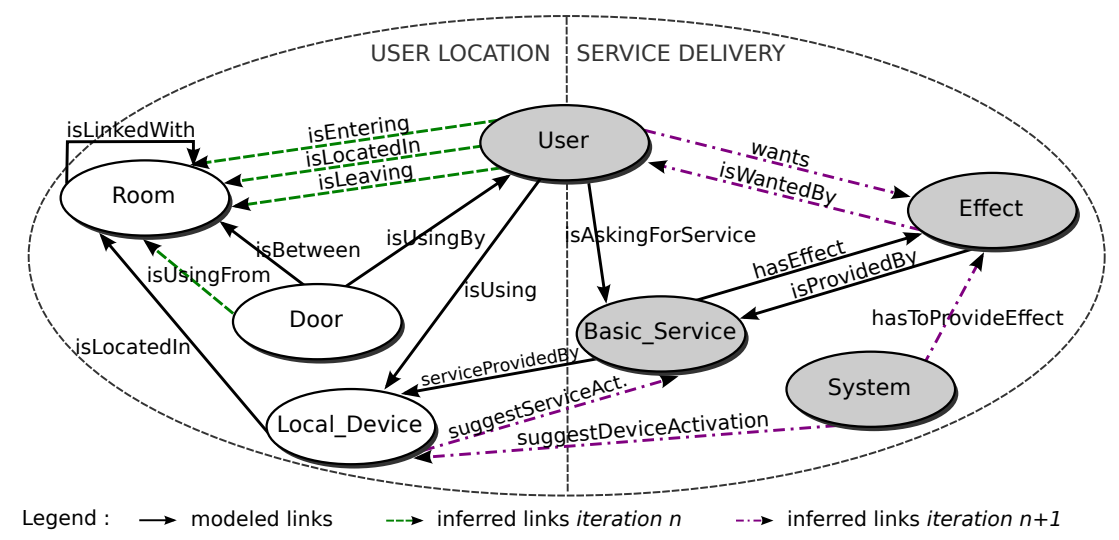

Fig. 1. Ontology (excerpts): user location (left side) and service delivery (right side).

\begin{tabular}{|c|c|c|}
\hline $\mathbf{N}^{\circ}$ & Name & SWRL expression (Semantic Web Rule Language) \\
\hline 1 & isLocatedIn & $\begin{array}{l}\text { LocalDevice(?d), Room(?r), User(?u), isLocatedIn(?d, ?r), isUsing(?u, ?d) } \\
\rightarrow \text { isLocatedIn(?u, ?r) }\end{array}$ \\
\hline 2 & isLeaving & $\begin{array}{l}\text { Door(?d), Room(?r), User(?u), isUsing(?u, ?d), isUsingFrom(?d, ?r) } \rightarrow \\
\text { isLeaving(?u, ?r) }\end{array}$ \\
\hline 3 & isEntering & $\begin{array}{l}\text { Door(?d), Room(?r1), Room(?r2), User(?u), isBetween(?d, ?r1), isBe- } \\
\text { tween(?d, ?r2), isLeaving(?u, ?r1), isLinkedWith(?r1, ?r2) } \rightarrow \text { isEnter- } \\
\text { ing(?u, ?r2) }\end{array}$ \\
\hline 4 & wants & $\begin{array}{l}\text { BasicService(?b), Effect(?e), User(?u), hasEffect(?b, ?e), isAskingForSer- } \\
\text { vice }(? \mathrm{u}, ? \mathrm{~b}) \rightarrow \text { wants }(? \mathrm{u}, ? \mathrm{e})\end{array}$ \\
\hline 5 & $\begin{array}{l}\text { suggestDevice- } \\
\text { Activation }\end{array}$ & $\begin{array}{l}\text { BasicService(?b), Device(?d), Effect(?e), Room(?r), System(?s), hasTo- } \\
\text { Provide(?s, ?e), isLocatedIn(?d, ?r), isProvidedBy(?e, ?b), isWantedIn(?e, } \\
\text { ?r), serviceProvidedBy(?b, ?d) } \rightarrow \text { suggestDeviceActivation(?s, ?d) }\end{array}$ \\
\hline 6 & $\begin{array}{l}\text { suggestService- } \\
\text { Activation }\end{array}$ & $\begin{array}{l}\text { BasicService(?b), Device(?d), Effect(?e), Room(?r), System(?s), hasTo- } \\
\text { Provide(?s, ?e), isLocatedIn(?d, ?r), isProvidedBy(?e, ?b), isWantedIn(?e, } \\
\text { ?r), serviceProvidedBy(?b, ?d) } \rightarrow \text { suggestServiceActivation(?d, ?b) }\end{array}$ \\
\hline
\end{tabular}


Given the user location, it is then possible to deliver services based on the effect concept: when a user asks for a service, he knows that this activation will have an effect on his environment. Basic services are linked with effect (Luminosity, Darkness, Heat, Quietness, etc.) through the hasEffect property (cf figure 1, right side). The user intention can thus be inferred from this kind of property. Let's consider the rule 4 from table 1: the user ? $u$ who is asking for a basic service ?b, wants the effect ?e associated with it.

Coupling the user intention with location information leads to service delivery. A user who wants luminosity (e.g. wants(User1, Luminosity)), and who enters a room will be offered services allowing to increase luminosity in the incoming room. Service delivery is based on suggestDeviceActivation and SuggestServiceActivation, applied respectively on System and Device (cf rules 5 and 6 from table 1). The design of such an ontology has to be flexible to adapt to user needs and evolving environments. On the prospect of a large scale service deployment, we choose not to do this complex modeling task by hand, but to automate it by means of a model-driven process.

\section{A model-driven architecture for ontology design}

In the context of ambient assisted living, we strongly believe that, in addition to the home automation expert, a non-expert - but someone who is able to properly consider the context of living with a disability (e.g. an occupational therapist, a family member) - must be included in the design process. We previously developped in [10] a model-driven flow for the design of assistive home automation systems. Platform independent modeling (PIM) are separated from platform specific modeling $(P S M)^{2}$, so that non-expert designers can focus on specification rather than on implementation. Using a domain specific language, they can model both environment and interactions to define respectively the context of use and the user needs, before automatically generating the control code ${ }^{3}$.

In this paper, we propose to automatically generate an ontology with the same domain specific language. Figure 2 shows the supervision module that consists of several steps integrated in the existing design flow. In step 1', relevant model concepts defined by this latter at PIM level are transformed into ontological concepts ( $P S M$ level). They have some basic information such as the layout of housing, devices parameters, but also services defined to consider user needs. Step 2' is about generating a knowledge base conformed to $O W L$, the most commonly used ontology language. The joint use of a model-driven architecture and ontology ${ }^{4}$ makes sense to benefit from their respective advantages, thus leading to the definition of a complete control and supervision system for smart home. MDA and languages derived from this paradigm offer few means to formally interpret the semantic of models at runtime. On the contrary, ontologies

\footnotetext{
${ }^{2}$ In accordance to the Model-Driven Architecture (MDA) specification [7].

${ }^{3}$ Experiments have been conducted to validate the assumption made above.

${ }^{4}$ The Ontology Definition Metamodel [8] is a specification to unify MDA and Ontology, making OWL conformed with the three modeling levels of the MDA specification.
} 


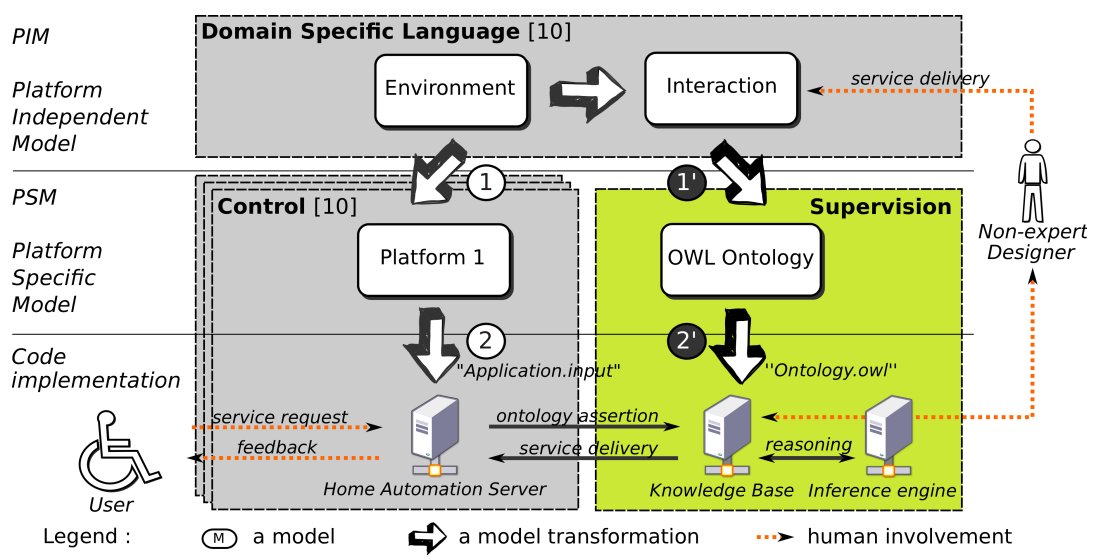

Fig. 2. User-centered design flow for the control and the supervision of smart home

make knowledge machine-understandable, by formalizing and by making explicit the semantic relations between concepts.

The implementation of our ontology as a non-intrusive supervision model is illustrated as follows. When the user asks for a service, it is first processed by the home automation server, before sending him back its execution result. The service request is also translated into an assertion in the ontology that represents and shares context information coming from various home automation platforms. Then, an inference engine has to interpret this assertion and to enrich the knowledge base with i) monitoring information that an home automation non-expert will be able to analyze to define new rules or new services and ii) service delivery on the user interface based on information previously inferred.

\section{Case study}

To validate our approach, a person tracking application for monitoring and for dynamic service delivery is presented in this section. This case study is based on a real monitoring dataset recorded in the Domus smart home $[11,12]$ from the University of Sherbrooke (cf figure 3). It consists of data coming from sensors. Since our aim is to avoid the use of a maximum of sensors, we have converted some of these sensors logs by user requests on devices (cf table 2) when such devices can be remotely controlled ${ }^{5}$. We consider an excerpt of activities performed in day 2 by user 1 [13].

Step 1: The user (user1) switches on the bedroom light: "[Log 1]: Light5105.SwitchON" ( $\log 1$ from table 2). This $\log$ is introduced in the ontol-

\footnotetext{
${ }^{5}$ As an example, Fl01 is a flow meter mounted on the cold water tap of the kitchen
} sink. It sends an event when the tap is opened but cannot be remotly controled 


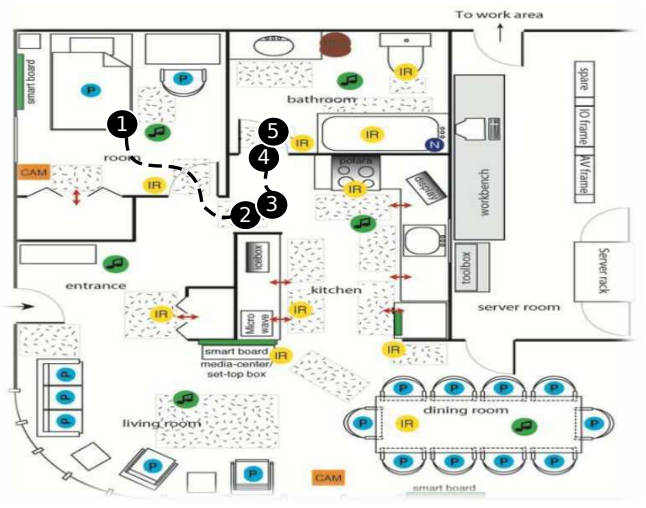

Fig. 3. A person tracking scenario in the Domus smart home, from [13].

\begin{tabular}{|c|c|c|c|c|c|c|}
\hline $\mathbf{N}^{\circ}$ & & Time & ID & Name & Location & Service/State \\
\hline 1 & * & $8: 56: 17$ & 5105 & Light & Bedroom & SwitchON \\
\hline 2 & * & $8: 56: 26$ & 0215 & Door & Bedroom & Open \\
\hline 3 & * & $8: 56: 31$ & 5101 & Light & Kitchen & SwitchON \\
\hline 4 & * & $8: 56: 35$ & 5105 & Light & Bedroom & SwitchOFF \\
\hline 5 & * & $8: 56: 39$ & 0215 & Door & Bedroom & Close \\
\hline 6 & * & $8: 56: 44$ & 0216 & Door & Bathroom & Open \\
\hline 7 & * & $8: 56: 46$ & 5106 & Light & Bathroom & SwitchON \\
\hline 8 & o & $8: 56: 51$ & Fl01 & T.ColdWater & Bathroom & Open state \\
\hline 9 & o & $8: 56: 54$ & Fl01 & T.ColdWater & Bathroom & Close state \\
\hline 10 & o & $8: 57: 09$ & IR01 & Infrared & KitchenSink & Close state \\
\hline 11 & o & $8: 57: 12$ & IR02 & Infrared & KitchenOven & Close state \\
\hline 12 & * & $8: 57: 12$ & 5106 & Light & Bathroom & SwitchOFF \\
\hline
\end{tabular}

Table 2. Dataset (excerpt) of series $1 /$ user $1 /$ day 2 considering devices activation $\left(*\right.$ : logs coming from user requests $/{ }^{\circ}$ : logs coming from sensors).

ogy through the following assertions ${ }^{6}$ : "[Assertion 1]: User1 isAskingForService SwitchON" and "[Assertion 2]: User1 isUsing Light5105". Knowing that the ontology defines Light5105 as a local activation resource located in Bedroom, and that this latter has a Switch $O N$ basic service with Luminosity effect, rules 1 and 4 from table 1 can be applied. The inference engine execution allows then to lead to the following inferences: [Inference 1]: User1 isLocatedIn Bedroom and [Inference 2]: User1 wants Luminosity.

Step 2: The user now wants to go to the bathroom: after opening the bedroom door, he switches on the kitchen light: "[Log 3]: Light5101.SwitchON". Since this light is considered as a local activation resource, the following inferences are realized: [Inference 3]: User1 isLocatedIn Kitchen and [Inference 4]: User1 wants Luminosity.

\footnotetext{
${ }^{6}$ Each home automation log is divided into isAskingForService and is Using properties.
} 
Step 3: The system proposes to switch off the bedroom light. This proposition is based on the inverse effect property (e.g. Luminosity isInverseOf Darkness). The user being located in the kitchen, the system proposes to switch off lights in other rooms. In this scenario, this service is accepted by the user: "[Log 4]: Light5105.SwitchOFF".

Step 4: To go into the bathroom, he asks for the door opening: "[Log 6]: Door0216.Open". Considering this door linked with exactly two rooms with the isBetween property, rules 2 and 3 of table 1 can be applied. The user-door interaction allows the inference engine to extract the following information: [Inference 5]: User1 isEntering Bathroom and [Inference 6]: User1 isLeaving Kitchen.

Step 5: Finally, the system proposes him to switch on the bathroom light $(\log 7)$, from user intention and location previously inferred. These inferences are made possible trough rules 5 and 6 from table 1 . Later, with the location information from sensor IR01, the system proposes him to switch off the bathroom light (as in step 3). This proposition is accepted by the user: "[Log 12]: Light5106.SwitchOFF".

The Domus smart home is equipped with infrared movement detectors, pressure detectors, switches contacts, and flow meters. This case study shows that the automatic inference of the user location by our model should limit the use to $37.5 \%$ of the sensors that has to be deployed to ensure this person tracking application. Sensors that can be removed are essentially switches contacts replaced by user requests and infrared movement/pressure detectors replaced by logic inferences. This case study can be generalized to all situations where the user moves from room to room, and needs the same services throughout its path (e.g. listening to music, watching tv, etc.). In a context of severe disabilities without economic constraint, quality of services may be preferred. If we decide to keep all sensors from this case study, this approach can improve the system dependability by sensors information redundancy $\left(66.7 \%\right.$ in this case study $\left.{ }^{7}\right)$.

\section{Conclusion}

The considerable increase of dependent people brings out important needs of monitoring to ensure a safe and secure independent living. In this context, this paper has presented a non-intrusive supervision model based on ontology for smart home. The supervision of such systems is not based on sensors use, but on the semantic analysis of logs recorded each time the user asks for a service. An ontology with associated rules allows the inference engine to extract information usually sensored. Finally, a case study based on a real monitoring data set validated our approach through a person tracking application.

This case study voluntarily uses very few sensors. A logic inference engine has to deduce the user location, thus replacing the use of movement detectors. However, to achieve a complete monitoring system over time, this approach

\footnotetext{
$\overline{7}$ On table 2 , the information from 8 sensors over 12 can be replaced/supplemented by a service request (see logs with $*$ ).
} 
should consider both user feedback and sensors whose information can not be inferred from home automation logs: information coming from biomedical sensors (e.g. blood pressure, core body temperature, etc.) or accurate information meters (e.g. temperature, luminosity, etc.). These latters could also be translated in assertions in the supervision model. Nevertheless, this approach allows the use of sensors to be limited, leading to a less intrusive and less expensive solution. Furthermore, it can improve the system dependability by redundancy of sensors information, especially important in ambient assisted living. In both cases, this approach provides a solution that may be more acceptable.

Service delivery is also an interesting contribution to allow users faster and easier access to services from their control interfaces ${ }^{8}$. A full-scale deployment is planned in two apartments of Kerpape $\mathrm{MFRRC}^{9}$ to evaluate how it can assist patients in their everyday life.

\section{References}

1. United Nations, Department of Economic and Social Affairs, Population Division: World Population Ageing (2009)

2. Weiser, M.: Some computer science issues in ubiquitous computing. Communications of the ACM. 36, 75-84 (1993)

3. Yu, C.R., Wu, C.L., Lu, C.H., Fu, L.C.: Human localization via multi-cameras and floor sensors in smart home. In: IEEE SMC (2006)

4. Nourizadeh, S., Deroussent, C., Song, Y.Q., and al.: Medical and home automation sensor networks for senior citizens telehomecare. In: IEEE ICC Workshops (2009)

5. Long, S., Holder, L.: Using graphs to improve activity prediction in smart environments based on motion sensor data. In: ICOST (2011)

6. Truong, T.B.T., de Lamotte, F.F., Diguet, J.P., and al: Proactive remote healthcare based on multimedia and home automation services. In: IEEE CASE (2009)

7. Miller, J., Mukerji, J., and al.: Model Driven Architecture (MDA). Object Management Group, Draft Specification ormsc/2001-07-01 (2001)

8. Consulting, D.F. and Deere, J.: Ontology Definition Metamodel (2005)

9. Gruber, T.R., and al.: Toward principles for the design of ontologies used for knowledge sharing. International Journal of Human Computer Studies. 43, 907-928 (1995)

10. Allègre, W., Burger, T., Berruet, P.: Model-Driven Flow for Assistive Home Automation System Design. In: 18th IFAC World Congress (2011)

11. Kadouche, R., Pigot, H., Abdulrazak, B., Giroux, S.: Support Vector Machines for Inhabitant Identification in Smart Houses. Ubiquitous Intelligence and Computing. 83-95 (2010)

12. Chikhaoui, B., Wang, S., Pigot, H.: A New Algorithm Based on Sequential Pattern Mining for Person Identification in Ubiquitous Environments. In: KDD Workshop on Knowledge Discovery from Sensor Data. 19-28 (2010)

13. Domus Monitoring Dataset, http://domus.usherbrooke.ca/jeux-de-donnees/

\footnotetext{
${ }^{8}$ Disabled persons with severe motion impairment (e.g. tetraplegia) can only control their home automation system by means of an automatic scrolling interface. In such restrictive situations, the selection of the desired service is very time consuming.

${ }^{9}$ Kerpape Mutualistic Functional Reeducation and Rehabilitation Center.
} 